\title{
A quantitative study of perceptual vector analysis
}

\author{
KIT H. SHUM and GEORGE L. WOLFORD \\ Dartmouth College, Hanover, New Hampshire
}

\begin{abstract}
Building on the general notion of perceptual vector analysis (Johansson, 1950) and the more quantitative coding model (Restle, 1979), we propose a description of the stimulus based on a Fourier analysis of the motion stimulus. The Fourier description of the motion is more general than Restle's description, which is based on the concept of circular motion. In addition, two experiments are reported in which subjects were asked to make psychophysical judgments about the orbits of moving stimuli. Those judgments allowed us to examine quantitatively the effects of several variables on perceptual vector analysis.
\end{abstract}

About 30 years ago, Johansson (1950) first formulated an interpretation of motion perception which is called perceptual vector analysis. Essentially, the concept of perceptual vector analysis is based on the extraction of a common motion component, leaving behind the residuals. This way of characterizing the complex motion appears to be more consistent with the percept than one based on the absolute motions. By absolute motion, we mean the motion relative to some stationary point in the environment. When the motion stimulus consists of a number of moving point-lights, or dots, an observer does not generally see a collection of unrelated dots, each moving on its individual trajectory. Instead, the separate dots appear to be organized in some fashion, depending on the characteristics of their individual motions. To use a frequently cited example (Figure 1), extraction of the common components $\vec{a}, \vec{b}$, and $\overrightarrow{c_{x}}$ in (b) gives rise to the interpretation depicted in (c), which is what subjects generally report seeing.

However, this notion of a common vector is ambiguous. For example, with the display shown in Figure 1a, another possible vector solution is depicted in Figure 2 . Here, the motion vector $\vec{c}$ is left intact, but vectors $\vec{a}$ and $\vec{b}$ are analyzed into components $\vec{a}_{y}$ and $\overrightarrow{a_{w}}, \vec{b}_{y}$ and $\vec{b}_{w}$, respectively. The $w$ components, being identical to $\vec{c}$, can be extracted as the common vector, leaving the $y$ components, which are motions relative to $C$. (The subscripts $x$, $y$, and $w$ in Figures 1 and 2 denote directions in which vectors are pointing.) In fact, any arbitrary vector

This paper is based, in part, on a dissertation submitted by K. H. Shum in partial fulfillment of the doctoral requirements at Dartmouth College. The research was supported, in part, by NIMH Grant MH33179 awarded to the second author. We are grateful to Carol A. Fowler for her helpful advice during all phases of the research. Requests for reprints should be sent to George Wolford, Department of Psychology, Dartmouth College, Hanover, New Hampshire 03755. can be extracted (or subtracted) from $\vec{a}, \vec{b}$, and $\vec{c}$. In such a solution, the dots $\mathrm{A}, \mathrm{B}$, and $\mathrm{C}$ move relative to some imaginary point of reference whose motion is given by the arbitrary vector. Thus, an important thing is to explain why a particular "solution," for example the one depicted in Figure 1c, is preferred.

This ambiguity was amply recognized in studies subsequent to Johansson's early work (Börjesson \& von Hofsten, 1972; Proffitt, Cutting, \& Stier, 1979; Restle, 1979). For example, Börjesson and von Hofsten (1972) pointed out specifically that "a vector analysis can be carried out in an indefinite number of ways unless one or several guiding principles are introduced." They went on to propose as a rule of thumb that the "magnitude and direction of the common component motion is so determined that the sum of the relative residual motion vectors equals zero" (p. 263). On the other hand, Proffitt et al. (1979) proposed that the common component is determined by the motion of the center of gravity of the dots, and Restle (1979) used the concept of information reduction. It is not easy to integrate all these different proposals. First, all three proposals were formulated on (a)

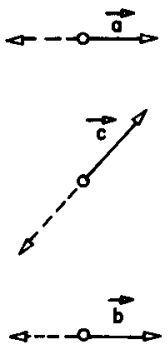

(b)

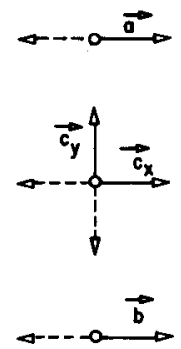

(c)

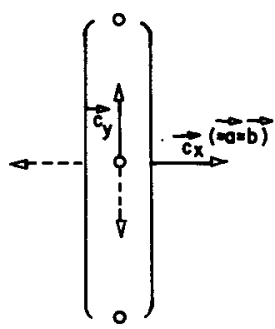

Figure 1. The interpretation proposed by Johangson (1974), based on resolving the diagonal vector $\vec{c}$ into $\overrightarrow{c_{x}}$ and $\overrightarrow{c_{y}}$. The subscripts $x$ and $y$ denote the direction of the vectors. 


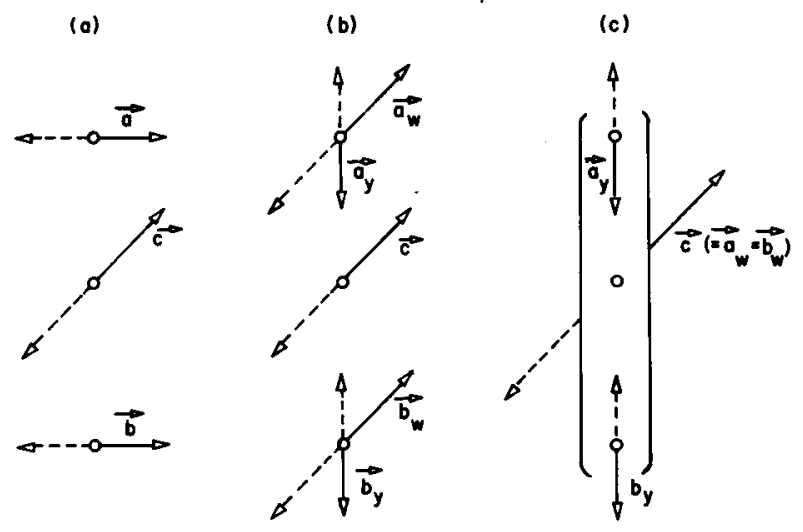

Figure 2. An alternative interpretation of the display in Figure 1. The subacripts $\boldsymbol{w}$ and $y$ indicate the direction of the vectors.

the basis of different sets of dynamic displays; and, second, the empirical data available for comparison among them is limited. Because of these limitations, the present understanding of the perceptual vector analysis phenomenon remains intuitive and qualitative, rather like the gestalt principles of perception. Lacking is a quantitative, yet general, language for describing the motion stimulus.

\section{Stimulus Specification}

Restle (1979) proposed a quantitative model for describing motion stimuli. Adapting Leeuwenberg's (1971) coding theory, Restle used a circular path, specified by five parameters, as the basic element in describing the displays studied by Johansson. Restle's formulation is limited in that it can describe only simple harmonic motions. ${ }^{1}$ All of Johansson's original displays were constructed of such motions. However, the basic idea behind Restle's model-to specify the motion stimulus in terms of simple scalar parameters-can be extended to encompass all conceivable motion paths. Our model represents one way of achieving this goal.

Before describing the model, we need to develop some basic concepts. One is the idea of a coordinate function. With the addition of a time dimension, any motion pattern in two dimensions can be represented by a three-dimensional graph. Consider the path of a single dot describing a circle. At any moment in time, the dot is at only one position on the plane. This possibility changes over time, such that the three-dimensional graph is a helix along the time dimension, represented by the z-axis in Figure 3a. In other words, we are dealing with a temporal function of the position of the moving dot. In Euclidean geometry, the position of a point on a plane can be specified by a pair of coordinates. Thus, the motion of the point in time might be defined by a pair of coordinate functions.

$$
\begin{aligned}
& x_{t}=f(t) \\
& y_{t}=g(t)
\end{aligned}
$$

For the circular path we have been talking about, these coordinate functions are pure sinusoids (Figures $3 b$ and $3 c)$, namely,

$$
\begin{aligned}
& x_{t}=r \cos (w t) \\
& y_{t}=r \sin (w t)
\end{aligned}
$$

where $r$ corresponds to the amplitude parameter of a sinusoid and is, in fact, the radius of the circle described by the moving dot, and $w$ is the frequency. The phase parameter of the sinusoid is disguised in the use of both sine and cosine. We can, instead, write:

$$
\begin{aligned}
& x_{t}=r \sin \left(w t+p_{1}\right) \\
& y_{t}=r \sin \left(w t+p_{2}\right)
\end{aligned}
$$

where now $p_{1}$ and $p_{2}$ denote the phase parameters, and equal 90 and $0 \mathrm{deg}$, respectively, for a circle.

In general, these coordinate functions may be combined to describe any form using a linear combination of sines and cosines, that is,

$$
h(t)=a_{0}+\sum_{n}\left[a_{n} \sin (n t)+b_{n} \cos (n t)\right]
$$

A sinusoid is completely defined by three scalar parameters, amplitude, frequency, and phase. Therefore, the coordinate functions, and hence the motion

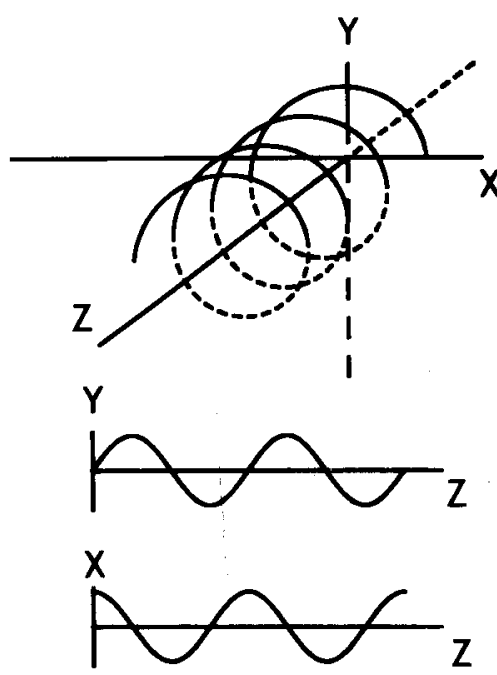

Figure 3. Three-dimensional graph of a two-dimensional circular path in time (a), and its projections onto the zy-plane (b) and $\mathrm{xx}$-plane (c), respectively. 
itself, can be represented by a set of scalar parameters, three for each sinusoid.

The power of this specification scheme is that any motion can be approximated by a series of Fourier components. For relatively simple motion patterns, it is possible to deduce what these functions and their Fourier components are. For very complex patterns, a technique known as cyclography may be used to obtain the coordinate functions, which can then be decomposed with computer algorithms (see Bernstein, 1967).

To assess the feasibility of this specification scheme, we did the converse by synthesizing motion displays from sinusoidal components. A program was written for a Terak 8510/a microcomputer which generates moving displays on a CRT screen. (The displays used in the experiments reported below were created in this way.) The core algorithm uses two orthogonal sets of sinusoidal components in calculating the successive positions along the trajectory of individual elements in the display (Shum, 1981). The displays created by this algorithm were of the type Cutting (1978) called dynamic point-light displays. Each consists of an arbitrary number of dots moving in their own paths, but as a whole specifies something like a walking person (Cutting, 1978) or a rolling wheel (Johansson, 1974; Proffitt et al., 1979).

We see the Fourier approach as a useful step in the quantification of perceptual vector analysis. The present descriptive model not only allows great flexibility in generating displays, but also permits quantitative measurement of the percept. In particular, the subjects in Experiment 2 responded interactively to the motion stimuli, through the computer system that generated the displays.

When the motion stimulus and its percept are described with such specificity, it becomes possible to look into the interplay among the individual motion vectors in an empirical and quantitative manner. Before proceeding to our experiments, we will examine some relevant empirical work.

\section{Experimental Studies of Perceptual Vector Analysis}

Johansson (1974) attempted to gather empirical support for the notion of perceptual vector analysis. Subjects were shown some form of trochoidal motion (the rolling wheel). The family of trochoidal motions can be interpreted as consisting of two motion components, a rotation and a linear translation. Varying the rate of rotation and the velocity of translation results in various trochoids. The experimental task was to adjust the two components to produce the trajectory of a rolling wheel. He reported that subjects did remarkably well, with a mean absolute percentage error of $\mathbf{1 8 \%}$. There was, however, an appreciable range among individual subjects, from $1 \%$ to $41 \%$. More will be said about individual differences later. More relevantly, it should be pointed out that the task might have little to do with extraction of common motion, because the task could have been performed by determining whether there was a retrograde loop in the trochoid or not. In other words, the task could have been accomplished simply by noting the discontinuity that exists between adjacent loops of a perfect cycloid.

Two subsequent studies (Hochberg \& Fallon, 1976; Mori, 1979) focused on the example of perceptual vector analysis described in Figure 1. Using an ingenious setup, Hochberg and Fallon (1976) obtained a quantitative measure of the perceptual vector analysis effect. Their results indicated that the perceived path of the middle dot was never judged to be truly vertical. Thus, they concluded that "the vectorextraction model must be phrased in more local terms" (Hochberg \& Fallon, 1976, p. 1083). In other words, the ideal solution as depicted in Figure $1 \mathrm{c}$ was not fully realized. Mori (1979) extended the technique to examine systematically the effect of spatial separation among the dots and found a linear relationship between the magnitude of the perceptual vector analysis effect, which ranged from $20 \%$ to $90 \%$, and the square root of the spatial separation.

It appears that the perceptual vector analysis phenomenon might be more appropriately conceptualized parametrically. The basic notion of perceptual vector analysis can be expressed in terms of motion relative to some moving reference. In the solution depicted in Figure 1c, the residual motion is, in fact, the motion of the middle dot, $\mathrm{C}$, relative to the moving pair A and B. This can be expressed explicitly as

$$
M_{p}=M_{a}-M_{f},
$$

where $M_{p}$ denotes the perceived motion, $M_{n}$, the given absolute motion, and $\mathbf{M}_{f}$, the motion of the frame of reference. The empirical results of Hochberg and Fallon (1976) and Mori (1979) evidently did not fit into this ideal model; but it is possible, instead, to conceive of an imperfect extraction of common motion without giving up the intuitively appealing idea put forth by Johansson. In other words, Expression 8 can be modified as follows:

$$
M_{p}=M_{a}-k M_{f},
$$

where $\mathbf{k}$ specifies the proportion of common motion that is extracted. The interpretation of this parameter will be left until later. For the time being, it provides a convenient way to capture the essence of the perceptual vector analysis phenomenon in a quantitative manner, as well as to interpret the experimental results to be reported below. 


\section{EXPERIMENT 1}

The first experiment was designed to establish the feasibility of using a psychophysical paradigm to arrive at a quantitative appraisal of the vector analytic approach. Given a display of moving dots, subjects were asked to make quantitative judgments about the shape of the trajectory of specific dots to see if the data could be interpreted in terms of perceptual vector analysis.

\section{Method}

Subjects. The subjects were 18 students enrolled in an introductory psychology course at Dartmouth College. There were 13 males and 5 females. They received course credit for their participation.

Stimuli. The stimuli were a series of 12 visual displays. Each display consisted of three moving dots. The displays were similar to ones used by Johansson (1950) and Restle (1979). One dot described a counterclockwise elliptical path. This was created by combining a vertical and a horizontal sinusoidal component. Two flanking dots oscillated along a vertical straight line in simple harmonic motion (i.e., there was no horizontal component). There were six different paths for the middle dot, each one an ellipse with a different eccentricity. All six had the same width (i.e., the same amplitude for the horizontal component) of $\mathbf{4 0}$ arbitrary units, while their heights (i.e., vertical components) were 0,5 , $10,20,40$, and 80 , respectively. In other words, the path of the middle dot ranged from a horizontal straight line, through various relatively "flat" ellipses, to a full circle, and then a "tall" ellipse. The amplitude of the vertical oscillation of the flanking dots was a constant $\mathbf{4 0}$ units, but these dots moved either in-phase or out-of-phase with the middle dot, depending on whether their vertical displacements were in synchrony with the middle dot or not. The maximum extent of the display subtended a range from 2.8 to $5.6 \mathrm{deg}$ of visual angle in both the horizontal and the vertical direction, depending on the viewing distance (from 8 to $4 \mathrm{~m}$ ) for the subjects, who participated in groups.

These stimuli were selected because they allow for the determination of a psychophysical function and also because they are predicted (under perceptual vector analysis) to lead to a rather striking percept. Even though all of the elliptical trajectories are counterclockwise, we would predict several of the ellipses to be perceived as moving clockwise when the flanking dots are in phase. This prediction follows from Equation 9. For instance, if actual motion is 20 and flanking motion is $\mathbf{4 0}$, then the predicted percept is -20 if $k$ equals 1 . The negative value (or change of sign) leads to the direction reversal (Figure 4).

Apparatus. The displays were created on a Terak 8510/a microcomputer. The motion of the dots was generated through a Fourier synthesis of two sets of orthogonal sinusoidal components. The video output of the computer was fed into a video cassette recorder to obtain a copy of all the 12 displays in a random sequence, which was subsequently used in the experiment. In addition to the moving displays, a transparency was constructed, which contained an array of 30 static ellipses, ranging from a horizontal straight line through a perfect circle to a very narrow "tall" ellipse in small gradations. The array was created using a plotting device (Tektronix 4662). These ellipses were used as the standards in the psychophysical judgment task.

Procedure. There were two viewing conditions, dark and light, with nine subjects in each. It was surmised that in the dark condition, the elimination of other visible objects that might conceivably serve as reference cues would maximize the perceptual vector analysis effect.

The subjects were tested in groups. First, they were shown the initial display and asked to write a brief description of it. This was to ensure that the percept of a middle dot moving round and
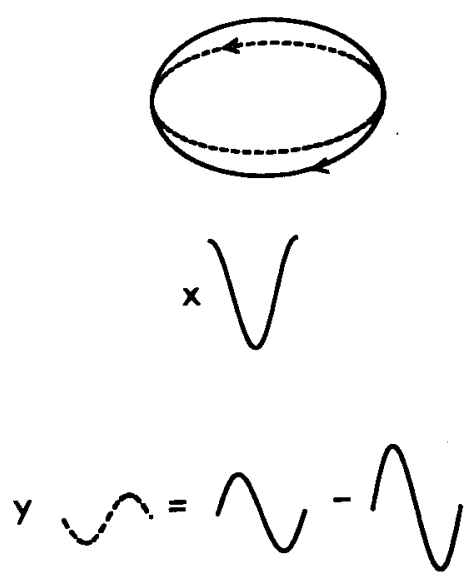

Figure 4. Resultant relative motion predicted by Equation 9 when the vertical components of the middle and flanking dots are in phase.

round between two other dots in concerted motion was naturally experienced. Then the static array of 30 ellipses was shown on a screen by an overhead projector. Each of the ellipses was denoted by a number. The subjects were told that their task, after watching each display, was to make a judgment of the eccentricity of the path of the middle dot by writing down the number associated with the appropriate ellipse in the array and also to specify the direction of the elliptical path (clockwise or counterclockwise). Then the series of motion displays was shown one after the other, alternating with the array of ellipses for the judgment task. In the dark condition, all the lights in the room were turned off. The light from the overhead projector at the end of each trial was sufficient for the subjects to write down their responses. In the light condition, the lights were left on all through the experimental session. The brightness/contrast of the video monitor was adjusted so that in both conditions the trail of the decaying phosphor was negligible.

\section{Results and Discussion}

The judgment data were translated into perceived amplitudes of the vertical motion component of the middle dot. Since the horizontal component was always the same, the amplitude of the vertical component directly reflects the shape, in this case, the eccentricity of the elliptical paths. To see how well the data fit the expressed relationship in Equation 9, the perceived amplitudes were regressed on the physical ones. The correlation coefficients for the individual subjects were predominantly above 0.9 with one outlying 0.56 . As predicted, the average subject in the dark condition saw the flattest four ellipses as travelling clockwise when the flanking motion was inphase. The slope and intercept estimates of the individual regression fits were subjected to analysis of variance tests, with two factors: lighting condition and the phase relationship between the middle dot and the flanking dots. According to perceptual vector analysis, the effect of these variables should show up in the intercept estimates.

No predictions follow from perceptual vector analysis concerning the slope estimates. To some ex- 
tent, the slopes represent the subjects' ability to judge the shapes of ellipses as a function of changing ratios of width to height. For the slope estimates, none of the main effects was significant at the .05 level. There was, however, a significant interaction, with $F(1,16)$ $=5.94, p=.026$. This seemed to indicate that the psychophysical functions for judging ellipticity was consistent among subjects. The interaction effect might be attributed to a ceiling effect.

On the basis of the reasoning developed earlier that led to Equation 9, the intercept estimates should give us some measure of the perceptual vector analysis effect. In particular, the extent to which the value of the intercept estimates approached the amplitude of the flanking dots (the reference in this case) will reflect how closely the percept was predicted by the relative motion. At one extreme, if the intercepts were identical to the amplitude (of the motion) of the frame of reference, the observer would be perceiving relative motion per se (i.e., an intercept of +40 for in-phase and $\mathbf{- 4 0}$ for out-of-phase); at the other extreme, a zero intercept would indicate that the observer was perceiving the absolute motion of the middle dot. Table 1 gives the mean intercept estimates.

Since the amplitude of the motion of the flanking dots was held at a constant of $\mathbf{4 0}$ arbitrary units, it is clear that the imperfect nature of the effect noted in Hochberg and Fallon (1976) and Mori (1979) was replicated. In terms of Equation 9, subjects in the present experiment appear to be subtracting out about $75 \%$ of the motion of the frame of reference from the absolute motion of the target in the dark condition; but this percentage falls to about $15 \%$ in the light condition. It should be pointed out that in the out-of-phase condition, subjects appeared to be adding instead of subtracting. This is because a difference in phase of $180 \mathrm{deg}$ is equivalent to a change in the sign of the amplitude parameter. In short, the addition was the result of a double negative in Equation 9, with the out-of-phase factor represented by negative amplitudes. From the analysis of variance of intercept values, the main effect of phase and the interaction of phase and lighting were statistically significant $[F(1,16)=157.81$ and $F(1,16)=39.74$, respectively, with probabilities approaching zero]. The main effect of lighting condition was not signif-

Table 1

Mean Intercepts, Slopes, and Estimates of $K$ for Experiment 1

\begin{tabular}{llrrc}
\hline & Condition & Intercept & \multicolumn{1}{c}{ K } & Slope \\
\hline \multirow{3}{*}{ Dark } & In-Phase & -29.93 & .75 & .94 \\
& Out-of-Phase & 31.63 & .79 & .71 \\
\multirow{2}{*}{ Light } & In-Phase & -5.10 & .13 & .89 \\
& Out-of-Phase & 8.63 & .22 & .99 \\
\hline
\end{tabular}

icant because the intercept values cancel out when averaged over phase. Even though there is a strong main effect of phase, it should be stressed that the data from both phases are handled well by a single equation with only a change of sign. The main effect of lighting conditions is reflected by different values of $k$ in that same equation. Thus, the parametric conceptualization of Equation 9 summarizes the results of the present experiment satisfactorily, with the parameter $\mathbf{k}$ capturing the quantitative gradations of the perceptual vector analysis effect.

The observed difference in the value of $k, .75$ vs. .15 , in the two viewing conditions may be interpreted as reflecting the amount of competing reference cues present. In the light condition, the availability of other visible objects, for example, furniture and the surface of the monitor screen, apparently permitted the subjects to perceive the absolute motion. In the dark condition, the absence of other cues led the subjects to use the moving flanking dots as the reference, favoring the perception of relative motion.

Finally, it was pointed out earlier that Johansson (1974) noted a considerable range of individual differences in degree of common motion extraction, in fact a range of $40 \%$. In the present parametric model, such individual differences can easily be subsumed under the same parameter $k$, representing the individual biases toward the perception of absolute vs. relative motion. During the experimental session, subjects were also given a version of the embeddedfigure test (for more on the embedded-figure test and the dimension of field dependence/field independence in "cognitive style," see Witkin, Lewis, \& Herzman 1954). The data were informal, and no attempt has been made to establish the standing of the scores of the subjects relative to the general population norm. But when the test scores were compared with the estimated values of $\mathbf{k}$ for individual subjects, a Spearman's rank correlation yielded a significant coefficient of .71 for the light condition. The correlation in the dark condition was .08 . This is consistent with the notion that when all the subject had available were the target and the two flanking dots, a boundary condition was approached, in which individual differences in cognitive style were masked by the more potent stimulus conditions.

\section{EXPERIMENT 2}

Watching the displays in Experiment 1, we noticed that the perceptual vector analysis effect was particularly pronounced when the target dot neared one of the flanking ones.

This should come as no surprise, in view of the data of Mori (1979), the adjacency principle espoused by Gogel $(1974,1978)$, and the proposed role of proximity in Ullman's (1979) correspondence formu- 
lation of motion perception. In fact, it suggested a situation in which the observed effect of perceptual vector analysis might be enhanced. Thus, in the experiment to be reported below, the pair of flanking dots was replaced by a square frame. Since at any point in the trajectory the target dot would be relatively close to the surrounding frame, it was expected that the value of the $k$ parameter might increase.

Since the amplitude of the frame in Experiment 1 was either plus or minus 40 , it was not possible to tell whether there was any differential effect of the amplitude of the motion of the frame. In a recent paper on induced movement, Rock, Auster, Schiffman, and Wheeler (1980) suggested that when the displacement of the inducing frame was subthreshold, the effect of the induced movement was more complete, in the sense that a greater portion of the movement of the frame would be transferred to the target. The present experiment employed six different levels of frame amplitude. The amplitude, in arbitrary units, of the vertical displacement of the square frame could take on one of five possible values: $-30,-10$, 0,10 , and 30 . (The positive and negative values corresponded to a phase difference of 180 , as pointed out earlier.) The zero amplitude was included as a baseline in which the square frame was stationary. In addition, a sixth condition, denoted by $\mathrm{X}$, was included in which there was no frame, that is, only the target dot was shown. This second control condition was an attempt to evaluate the effect of the frame per se, without movement, on the judgment task.

There was also a modification in the nature of response. In Experiment 1, subjects were asked to choose among 30 standard ellipses with graded eccentricities, which were created by fixing the length of one axis while changing that of the other axis in equal steps. This use of an arbitrary step size imposed a limit on how fine a discrimination the subject could make. The question was how to strike a balance between accuracy and a manageable number of choices. In the present experiment, this problem was solved by having the subject vary the shape of the elliptical path directly by push-button controls until the desired path was obtained. In this way, the accuracy could be increased while eliminating the need to introduce static traces of whole ellipses into the situation.

\section{Method}

Subjects. Fifteen subjects were recruited from the Dartmouth student community. There were 12 females and 3 males. They were paid $\$ 3$ for participating in the experiment, which lasted about $45 \mathrm{~min}$.

Stimuli and Apparatus. The two flanking dots were replaced by a surrounding square frame, which moved at one of five possible amplitudes, including zero. Two designated keys on the key- board of the computer allowed the subject to manipulate the vertical amplitude of the dot. The displays subtended a visual angle of 2.4 horizontally and a maximum of 3.6 vertically, depending on the amplitude of the motion of the square frame, at a viewing distance of $3 \mathrm{~m}$ for all subjects.

Procedure. Subjects were tested individually, in the dark. The experiment consisted of six blocks of seven trials each, with each block corresponding to a particular level of the frame factor. Subjects were asked to make the orbit of the target dot into different shapes. There were four basic target shapes: a circle, a 2:1 ellipse (in which the width is twice the height), a 4:1 ellipse, and a straight line (i.e., an ellipse with zero height). Except for the straight line, the subjects were asked to produce both a clockwise and a counterclockwise version of each shape, making a total of seven different orbits. No standards were provided, subjects were instead asked to produce shapes such that the width appeared to bear a certain ratio to the height. The order of the shapes, as well as the level of the frame factor, was randomized. Subjects made all their judgments in the dark. The surface of the monitor screen was not discernible, even though the blackout procedure was not perfect.

Each trial began with the target dot moving in an elliptical path with a vertical amplitude randomly chosen within the range plus and minus 40 . The experimenter then instructed the subject to produce a specific path. This was accomplished by pressing one of two keys which changed the shape of the path in opposite directions in steps of one arbitrary unit. Subjects were encouraged to overshoot the target shape and then backtrack in order to converge on the desired path. This prompting was designed to counteract or eliminate any systematic anticipation effects that might occur.

\section{Results and Discussion}

The subject-produced amplitudes were recorded for each trial. These were recorded as the absolute given motion, while the target shapes (e.g., 2:1 counterclockwise, which translated to an amplitude of 20) were taken to be the perceived amplitudes. As in Experiment 1, a linear regression produced slope and intercept estimates for individual subjects, which were then subjected to an analysis of variance. These regression fits are shown in Figure 5. The amplitude of the motion of the frame did not affect the slope estimates $[F(5,70)=0.54]$, but had a strong effect on the intercept estimates $[F(5,70)=173.1, p=0]$. The

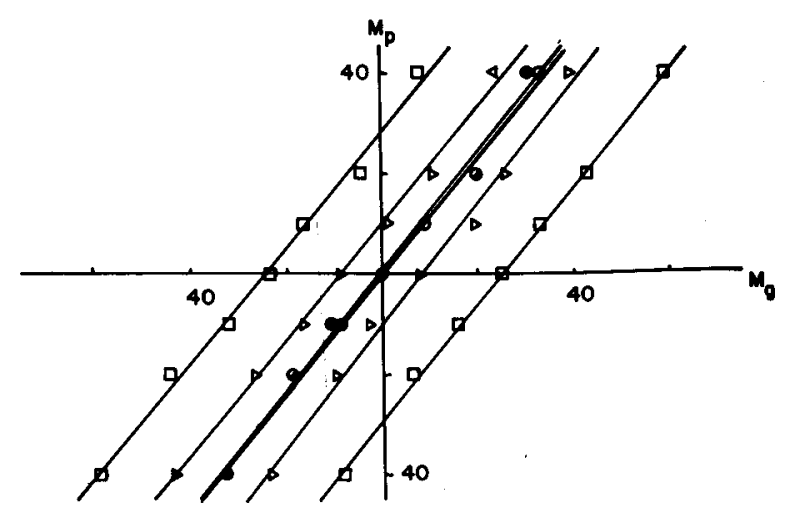

Figure 5. Mean regression lines for Experiment 2. 
Table 2

Mean Intercepts, Slopes, and Estimates of $\mathrm{K}$ for Experiment 2

\begin{tabular}{crrr}
\hline $\begin{array}{c}\text { Amplitude } \\
\text { of Frame }\end{array}$ & Intercept & $\mathrm{K}$ & Slope \\
\hline 30 & -29.16 & .97 & 1.16 \\
10 & -10.01 & 1.00 & 1.21 \\
0 & -0.13 & & 1.17 \\
-10 & 9.54 & .95 & 1.15 \\
-30 & 28.27 & .94 & 1.14 \\
No Frame & 0.70 & & 1.18 \\
\hline
\end{tabular}

mean intercept estimates are given in Table 2. Another way of looking at the intercept estimates is in terms of the parameter $k$ in Equation 9. That is, the value of $k$ can be separately estimated for each of the frame amplitudes; and they came out to be about 0.96 for all the nonzero amplitude conditions (see Table 2). An analysis of variance was carried out on the estimates of $\mathbf{k}$ from the four conditions with nonzero frame amplitudes. The obtained value of $F(3,42)=$ 0.42 indicated no effect of frame amplitude on the percentage of frame motion extracted. In other words, subjects appeared to subtract out $95 \%$ or more of the motion of the frame in their percepts, independent of the amplitude of the frame's motion.

In summary, as predicted, the square frame was a more effective inducer of the perceptual vector analysis effect than the flanking dots, and in the present viewing condition resulted in the perception of relative motion ( $k=0.96$ in this case). The finding that there was no differential effect of frame amplitude should be interpreted with caution. First, to the extent that the motion of the frame was not subthreshold, the suggestion of Rock et al. (1980) might yet be confirmed. Second, the fact that $k$ approached unity in general in the present setup might have created a ceiling effect, which implies that a different viewing condition, similar to the light condition in Experiment 1 , might be more appropriate in addressing Rock et al.'s (1980) suggestion.

\section{GENERAL DISCUSSION}

The first objective of the present study was to come up with an explicit description of the motion stimulus. Using the idea of Fourier decomposition, the model presented here specifies any motion in a two-dimensional (frontal) plane in terms of two orthogonal sets of sinusoidal components. The scheme can be easily generalized to the three-dimensional case using three mutually orthogonal sets of components. But, for simplicity's sake, we have limited ourselves to two dimensions. The advantage of such a descriptive model lies not just in the ability to talk about the motion stimulus in precise, though somewhat arbitrary terms (Footnote 1), but also in the ease with which motion displays can be generated with the aid of computer graphics or electronic circuitry.

The use of sinusoidal components also minimizes the degree of mathematical sophistication needed to cope with the kinematics: only scalar parameters (frequency, amplitude, and phase) are involved. Nevertheless, it is sufficiently powerful to approximate any motion patterns.

We have also demonstrated a viable experimental paradigm for quantifying the perceptual vector analysis effect. The results obtained are not only interesting in their own right, but also, more importantly,suggest a psychophysical procedure to examine the relationship between the objectively given motion, on the one hand, and its perceptual counterpart, on the other. The provocative work of Gunnar Johansson can now be conceptualized in more explicit (and quantitative) terms. In particular, the question of how the motion vectors should be partitioned might be addressed through empirical means. That is, with quantitative data, it will be possible to compare the different formulations of how perceptual vector analysis is performed. In any case, some of the effects observed were rather powerful, namely that, under favorable conditions (Experiment 2 ), subjects were essentially exhibiting the perfect perceptual vector analysis effect $(k=.95)$.

We observed large individual differences in estimates of the parameter $k$. Johansson (1974) also noted such individual differences. These differences appear to reflect the extent to which subjects use stationary objects in the environment as their frame of reference rather than the flanking dots. The choice of frame of reference does not appear to be strongly subject to intentional control. When we removed information about objects in the environment (dark condition), the estimates of $\mathbf{k}$ were far less variable.

\section{REFERENCE NOTE}

1. Shum, K. H. A quantitative study of perceptual vector analysis. Unpublished doctoral dissertation, Dartmouth College, 1980.

\section{REFERENCES}

Bernstein, N. The co-ordination and regulation of movements. London: Pergamon, 1967.

Börjesson, E., \& von Horsten, C. Spatial determinants of depth perception in two-dot motion patterns. Perception \& Psychophysics, 1972, 11, 263-268.

CutTing, J. E. A program to generate synthetic walkers as dynamic point-light displays. Behavior Research Methods \& Instrumentation, 1978, 10, 91-94.

Goges, W. C. Relative motion and the adjacency principle. Quarterly Journal of Experimental Psychology, 1974, 14, 425-437.

Goges, W. C. The adjacency principle in visual perception. Scientific American, 1978, 238, 126-137.

Hochberg, J., \& Fallon, P. Perceptual analysis of moving patterns. Science, 1976, 194, 1081-1083. 
Johansson, G. Configurations in event perception: An experimental study. Stockholm: Almqvist \& Wiksells, 1950.

JoHANSson, G. Vector analysis in visual perception of rolling motion: A quantitative approach. Psychologische Forschung, 1974, 36, 311-319.

Leeuwenberg, E.L.J. A perceptual coding language for visual and auditory patterns. American Journal of Psychology, 1971, 84, 307-349.

MorI, T. Relative locations among moving spots and visual vector analysis. Perceptual \& Motor Skills, 1979, 48, 587-592.

Proffitt, D. R., Cutting, J. E., \& Stier, D. M. Perception of wheel-generated motions. Journal of Experimental Psychology: Human Perception and Performance, 1979, 5, 289 302.

RESTLE, F. Coding theory of the perception of motion configurations. Psychological Review, 1979, 86, 1-24.

Rock, I., Auster, M., Schiffman, M., \& Wheeler, D. Induced movement based on subtraction of motion from the inducing object. Journal of Experimental Psychology: Human Perception and Performance, 1980, 6, 391-403.

ShUm, K. H. An algorithm to generate dynamic point-light displays. Behavior Research Methods \& Instrumentation. 1981, 13, 377-378.
Tonn, J. T. Visual information about rigid and nonrigid motion: A geometric analysis. Journal of Experimental Psychology: Human Perception and Performance, 1982, 8, 238-252.

Ullman, S. The interpretation of visual motion. Cambridge, Mass: M.I.T. Press, 1979.

Witkin, H. A., Lewis, H. B., \& Herzman, M. Personality through perception. New York: Harper, 1954.

\section{NOTE}

1. Todd (1982) presents a similar analysis with respect to an elliptical trajectory, but with seven parameters instead of five. The only difference is that Restle (1979) used a canonical form, without considering the explicit physical location in two-dimensional space, while Todd (1982) used two more parameters to designate the position of the elliptical path. (This was also pointed out in Shum, Note 1, p. 15.)

(Manuscript received February 18, 1983; accepted for publication March 7, 1983.) 